\title{
Towards a general solution of the Hamiltonian constraints of General Relativity
}

\author{
A. Tiembld a and R. Tresguerredi \\ Instituto de Matemáticas y Física Fundamental \\ Consejo Superior de Investigaciones Cientificas \\ Serrano 113 bis, 28006 Madrid, SPAIN
}

(Dated: December 21, 2018)

\begin{abstract}
The present work has a double aim. On the one hand we call attention on the relationship existing between the Ashtekar formalism and other gauge-theoretical approaches to gravity, in particular the Poincaré Gauge Theory. On the other hand we study two kinds of solutions for the constraints of General Relativity, consisting of two mutually independent parts, namely a general three-metricdependent contribution to the extrinsic curvature $K_{a b}$ in terms of the Cotton-York tensor, and besides it further metric independent contributions, which we analyze in particular in the presence of isotropic three-metrics.
\end{abstract}

PACS numbers: 04.20.Cv, 04.20.Jb

\section{INTRODUCTION}

In what follows we are mainly concerned with the search for a solution of the Hamiltonian constraints of General Relativity (GR). However, incidentally we are also interested in showing the underlying linkage existing between the Ashtekar formalism and the Hamiltonian Poincaré Gauge Theory (PGT) discussed by us in previous works 1] 2]. In our view, the internal $S O(3)$ symmetry affecting Ashtekar's variables [3] reveals to be the explicit manifestation of a wider gauge group. In order to corroborate this fact, we present the constraints of GR in different formulations, showing the bond between the PGT, the Ashtekar and the ADM ones 4].

After revising previous attempts to solve the constraints of GR, we derive our main result, consisting of equation (53), which provides a solution of both the Gauss law and the ADM momentum constraint (or Ashtekar's vector constraint) by expressing the exterior curvature in terms of the three-metric and its derivatives. Any other solution is defined up to this metric dependent contribution. We finalize discussing additional acceptable solutions irreducible to (53).

As a necessary reference for our discourse, let us first of all review the basics of the Ashtekar Hamiltonian formulation of gravity generalized by Barbero [5] 6] 7], where the fundamental dynamical variables are given by the canonically conjugate pair $\left(E_{I}^{a}, A_{a}^{I}\right)$. (In our notation, small latin letters $a, b \ldots$ of the beginning of the alphabet are assigned to the coordinates of the three-dimensional spatial slices resulting from a suitable foliation of spacetime, while capital letters $I, J \ldots$ of the middle of the alphabet display internal $S O(3)$ indices running from 1 to 3.) The first pair element consists of the densitized triads

$$
E_{I}^{a}:=e e_{I}^{a}
$$

*tiemblo@imaff.cfmac.csic.es

romualdo@imaff.cfmac.csic.es defined from the triad components $e_{I}^{a}$ on the spatial slices, with the corresponding determinant $e:=$ $\frac{1}{3 !} \epsilon^{a b c} \epsilon_{I J K} e_{a}^{I} e_{b}{ }^{J} e_{c}{ }^{K}$. The second pair element, that is the momentum conjugate to $E_{I}{ }^{a}$, is the $S O(3)$ connection

$$
A_{a}^{I}:=\Gamma_{a}^{I}(e)+\beta K_{a}^{I},
$$

constituted by two contributions, namely the triad compatible spin coefficients $\Gamma_{a}^{I}(e)$, whose explicit form is given in (3) with (4) below, and the extrinsic curvature tensor $K_{a}^{I}$ of the slices (with an index converted into a triad index), being both combined by means of the Barbero-Immirzi parameter $\beta$. Explicitly, the spin part of (2) is built from the triads $e_{I}{ }^{a}$ as

$$
\Gamma_{a}^{I}(e):=\frac{1}{2} \epsilon^{I J K} \hat{\Gamma}_{a J K}(e)
$$

with

$$
\hat{\Gamma}_{a J K}(e):=-e_{[J}^{b}\left(\partial_{a} e_{b K]}-\Gamma_{a b}^{c} e_{c K]}\right),
$$

see (A21), where $\Gamma_{a b}{ }^{c}$ stands for the holonomic spatial Christoffel symbol

$$
\Gamma_{a b}^{c}:=\frac{1}{2} q^{c d}\left(\partial_{a} q_{d b}+\partial_{b} q_{d a}-\partial_{d} q_{a b}\right)
$$

expressed in terms of the holonomic three-metric $q_{a b}:=$ $\delta_{I J} e_{a}^{I} e_{b}^{J}$ defined from the triads.

The constraints of General Relativity in terms of (1) and (2), as derived in the literature [5] [6] 7], are the weakly vanishing expressions

$$
\begin{aligned}
\mathcal{G}_{I} & :=\nabla_{a} E_{I}^{a} \approx 0 \\
\mathcal{V}_{a} & :=E_{I}{ }^{b} F_{a b}^{I} \approx 0 \\
\mathcal{S} & :=\epsilon_{I}{ }^{J} E_{J}{ }^{a} E_{K}{ }^{b} F_{a b}^{I} \\
-\frac{2\left(1+\beta^{2}\right)}{\beta^{2}} & E_{[I}^{a} E_{J]}^{b}\left(A_{a}^{I}-\Gamma_{a}^{I}\right)\left(A_{b}{ }^{J}-\Gamma_{b}{ }^{J}\right) \approx 0
\end{aligned}
$$


representing (6) the Gauss law, (7) the vector constraint and (8) the scalar constraint respectively, with both the covariant derivative

$$
\nabla_{a} E_{I}^{a}:=\partial_{a} E_{I}^{a}+\epsilon_{I J}{ }^{K} A_{a}^{J} E_{K}^{a},
$$

and the $S O(3)$ field strength

$$
F_{a b}^{I}:=2 \partial_{[a} A_{b]}^{I}+\epsilon^{I}{ }_{J K} A_{a}^{J} A_{b}^{K},
$$

built with the Barbero $S O(3)$ connection (2), being $\epsilon_{I J K}$ the $S O(3)$ Levi-Civita tensor. Since (6) - (8) are all of them first class constraints [8], they behave as generators of symmetries, corresponding (6) to the internal $S O(3)$ group, (7) to three-dimensional diffeomorphisms and (8) to reparametrization invariance.

\section{ASHTEKAR TYPE VARIABLES FROM PGT}

We start making a brief comment on the relationship existing between Ashtekar type formulations [9] 10] 11] 12] 13] and those alternative realizations of GR as a gauge theory -very close to standard Yang-Mills theories- mainly due to Hehl and coworkers [14 [15] 16 17] 18. The correspondence between both approaches was extensively studied by us in 2]. Here we summarize the basic points.

Hamiltonian treatments of GR based on any of the two mentioned gauge-theoretical versions of gravity reveal to be transformable into each other provided one identifies the Ashtekar variables with certain combinations of quantities taken from the alternative approach, as discussed below and shown in (111). The seemingly different symmetries involved -the $S O(3)$ internal Ashtekar symmetry versus, say, the local Poincaré symmetry of PGT - are conciliated by the fact that nonlinear realizations of groups allow to build actions being invariant under a given group but manifestly invariant only under a subgroup of the total gauge group. Accordingly, gravitational actions formulated exclusively in terms of $S O(3)$-symmetric quantities can be simultaneously invariant under a wider local spacetime symmetry not explicitly displayed.

Let us consider the nonlinear PGT building blocks presented in the Appendix. We are going to show how to get the Ashtekar reformulation of the corresponding gravitational equations; in particular of the Hamiltonian constraints. Since a homogeneous notation is needed in order to compare the Ashtekar equations with those built from the PGT quantities A8 -A11, we proceed to translate (6)-(8) into the language of exterior calculus ${ }^{1}$. The

\footnotetext{
${ }^{1}$ For what follows, an important observation is in order. Due to the fact that we will deal with constraints, one must have
}

Barbero-Ashtekar connection (2), rewritten as a 1-form $A^{I}=d x^{a} A_{a}^{I}$, becomes

$$
A^{I}:=\Gamma^{I}+\beta K^{I},
$$

where, in general, the Immirzi parameter $\beta$ is a complex number to be fixed. The link to PGT follows from identifying (11) as a combination of the real nonlinear PGT connection fields A10 and (A11), being $\Gamma^{I}$ the connection associated to the $S O(3)$ subgroup of the Lorentz group $^{2}$, while $K^{I}$ is the nonlinear connection of the boost subgroup of the Lorentz group. (Notice that this gaugetheoretical explanation of $K^{I}$ as a Poincaré quantity is compatible with its usual interpretation as the extrinsic curvature). According to A15 and A14, $\Gamma^{I}$ transforms as an $S O(3)$ connection and the nonlinear connection $K^{I}$ as an $S O(3)$ tensor respectively, so that the composite Ashtekar type variable (11) -in general a complex quantity- behaves as a modified $S O(3)$ connection in which both the $S O(3)$ part and and the boost part of the Lorentz connection of PGT are comprised.

The interpretation of the Ashtekar formalism in terms of Poincaré quantities is completed by identifying the coframes as the nonlinear translational connections (A8), (A9) ${ }^{3}$, being in particular (A9) taken to be indistinguishable from the triads built from the Ashtekar triad components $e_{a}{ }^{I}$ as $\vartheta^{I}:=d x^{a} e_{a}{ }^{I}$, see (1), and transforming as an $S O(3)$ covector, as shown in (A13). (In order to understand also the role played by the time component (A8), one should consider the whole four-dimensional formalism previous to the foliation. The interested reader is referred to our papers [1] 2].)

The field strength built from (11) is the 2 -form

$$
\begin{aligned}
F^{I} & :=d A^{I}+\frac{1}{2} \epsilon^{I}{ }_{J K} A^{J} \wedge A^{K} \\
& =\mathcal{R}^{I}+\frac{1}{2}\left(1+\beta^{2}\right) \epsilon^{I}{ }_{J K} K^{J} \wedge K^{K}+\beta \stackrel{\Gamma}{\nabla} K^{I},
\end{aligned}
$$

whose components as given by $F^{I}=\frac{1}{2} F_{a b}^{I} d x^{a} \wedge d x^{b}$ are identical with (10). In (12) we introduced the $S O(3)$ part of the Poincaré curvature (A17), defined as

$$
\mathcal{R}^{I}:=d \Gamma^{I}+\frac{1}{2} \epsilon^{I}{ }_{J K} \Gamma^{J} \wedge \Gamma^{K}-\frac{1}{2} \epsilon^{I}{ }_{J K} K^{J} \wedge K^{K},
$$

in mind that although we use the same notation as in the Appendix, where the quantities considered are previous to any foliation of spacetime, here we only consider the spatial parts of such quantities. The latter ones are defined on the spatial slices resulting from the foliation with respect to the time vector $\hat{e}_{0}=\frac{1}{N} \partial_{\tau}-N^{a} \partial_{a}$, having imposed the time gauge in such a way that the time component of the tetrads reduces to $\hat{\vartheta}^{0}=N d \tau$.

${ }^{2} \Gamma^{I}$ is expressible in terms of frame and coframe fields as $\Gamma^{I}(e)$ when torsion is absent.

3 Here we suppress the hat over the $S O(3)$ quantities present in the Appendix, where it is necessary in order to distinguish them from the Lorentz ones. 
as much as the boost part of such curvature, that is

$$
\stackrel{\Gamma}{\nabla} K^{I}:=d K^{I}+\epsilon_{J K}^{I} \Gamma^{J} \wedge K^{K} .
$$

In the following we simplify things by fixing $\beta=i$ in (11), so that the latter becomes a standard complex Ashtekar variable [5] [19] and (12) reduces to

$$
F^{I}=\mathcal{R}^{I}+i \stackrel{\Gamma}{\nabla} K^{I}
$$

We do not enter the discussion on the physical relevance of the value assigned to the Barbero-Immirzi parameter [5] 6] 19] 20] 21]. Let us merely mention that at the classical level Einstein's equations have the same physical content for any value of $\beta$, being different choices of such value related to each other by means of canonical transformations. The Immirzi quantization ambiguity concerns quantum gravity, where distinct phase spaces corresponding to different values of $\beta$ correspond to unitarily inequivalent quantum theories 21]. However, the possible role of $\beta$ as a fundamental constant in quantum gravity, to be measured empirically, seems to be not well understood 7]; and in any case our choice could be changed if necessary.

We end this section using (15) and of the triads (A9) to translate the constraints (6) - (8) into the language of exterior calculus. The Gauss constraint (6) can be rewritten as

$$
\mathcal{G}_{I}=e^{\#}\left(\nabla \eta_{I}\right) \approx 0
$$

in terms of the eta-basis 2-form defined as

$$
\eta_{I}:=\frac{1}{2 !} \epsilon_{I J K} \vartheta^{J} \wedge \vartheta^{K}=\frac{1}{2 !} \epsilon_{I J K} e_{a}^{J} e_{b}^{K} d x^{a} \wedge d x^{b} .
$$

The symbol \# stands for the Hodge dual in three dimensions; see for instance 1]. (In particular, (17) is the Hodge dual of the triad, that is $\eta_{I} \equiv{ }^{\#} \vartheta_{I}$.) On the other hand, the vector constraint (7) becomes

$$
d x^{a} \mathcal{V}_{a}:=d x^{a} E_{I}^{b} F_{a b}^{I}=e^{\#}\left(\vartheta_{I} \wedge^{\#} F^{I}\right) \approx 0
$$

where the three-dimensional Hodge dual of $F^{I}=$ $\left.\left.\frac{1}{2} F_{a b}^{I} d x^{a} \wedge d x^{b}=\frac{1}{2}\left(e_{K}\right\rfloor e_{J}\right\rfloor F^{I}\right) \vartheta^{J} \wedge \vartheta^{K}$ reads ${ }^{\#} F^{I}=$ $\left.\left.\frac{1}{2}\left(e_{K}\right\rfloor e_{J}\right\rfloor F^{I}\right) \epsilon^{J K}{ }_{L} \vartheta^{L}$. Analogously, the scalar constraint (8) (with the chosen value $\beta=i$ ) takes the form

$$
\mathcal{S}:=\epsilon_{I}^{J K} E_{J}{ }^{a} E_{K}{ }^{b} F_{a b}^{I}=2 e^{2 \#}\left(\vartheta_{I} \wedge F^{I}\right) \approx 0,
$$

where we made use of the fact that the Hodge dual of the three-form $d x^{a} \wedge d x^{b} \wedge d x^{c}$ is identical with the three-dimensional Levi-Civita tensor density $\eta^{a b c}$, that is $\#\left(d x^{a} \wedge d x^{b} \wedge d x^{c}\right)=\eta^{a b c}=\epsilon^{I J K} e_{I}^{a} e_{J}^{b} e_{K}^{c}$. In view of (16), (18) and (19), we can replace the constraints (6) -(8) by their exterior calculus formulation

$$
\begin{array}{rlr}
\nabla \eta_{I} \approx 0 & (\text { GAUSS }) \\
\vartheta_{I} \wedge{ }^{\#} F^{I} \approx 0 & (\text { VECTOR }) \\
\vartheta_{I} \wedge F^{I} \approx 0 & (\text { SCALAR })
\end{array}
$$

The tortuous way followed here to get the form (20)(22) of the constraints is justified by the attempt to make evident the relationship between PGT and the Ashtekar formalism. However, the interested reader is referred to [2], where we deduced such constraints directly from a Poincaré invariant gravitational action.

\section{PREVIOUS PARTIAL SOLUTIONS OF THE CONSTRAINTS}

A new solution for the system (20)- (22) -to be added to any other possible solution- will be studied in next section, fulfilling both the Gauss law (20) and the vector constraint (21), leaving only the scalar constraint (22) unsolved. The novelty of that solution, given by Eq. (153) below expressing the extrinsic curvature in terms of the three-metric, is emphasized by comparing it with previous results. Let us recall here in particular the one due to Capovilla, Dell and Jacobson [22], which formally solves the vector and scalar constraints (21) and (22) respectively, and the related Barbero proposal [23] solving the Gauss law (20) and the scalar constraint (22). We review both approaches in a simple reformulation adapted to the notation introduced in previous section.

In essence, the solution of the type proposed by Capovilla et al. 24] rests on a suitable form to depict the components of the field strength (15), namely

$$
F^{I}=\Psi^{I J} \eta_{J}
$$

with $\Psi^{I J}$ as a $3 \times 3$ complex matrix and $\eta_{I}$ as the 2 -form given by (17). The vector constraint (21) then turns into

$$
\vartheta_{I} \wedge{ }^{\#} F^{I}=\Psi^{I J} \vartheta_{I} \wedge \vartheta_{J} \approx 0
$$

while the scalar constraint (21) becomes

$$
\vartheta_{I} \wedge F^{I}=\Psi^{I J} \vartheta_{I} \wedge \eta_{J}=\Psi_{I}^{I} \eta \approx 0 .
$$

(In (25) we made use of the identity $\vartheta^{I} \wedge \eta_{J}=\delta_{J}^{I} \eta$, with $\eta:=\frac{1}{3 !} \epsilon_{I J K} \vartheta^{I} \wedge \vartheta^{J} \wedge \vartheta^{K}$ as the volume element.) From (24) and (25) one trivially reads out that both the vector and the scalar constraint are satisfied by any symmetric traceless matrix $\Psi^{I J}$. However, (20) is not automatically fulfilled. Instead, it gives rise to the condition

$$
\left[\nabla\left(\Psi^{-1}\right)_{I J}\right] \wedge F^{J} \approx 0
$$

involving three differential equations to be solved. This makes the solution of Capovilla et al. unsatisfactorily incomplete, not to speak about its rather formal character.

An alternative to the previous solution was proposed by Barbero [23]. Let us briefly revise it, suitably accommodated to our notation. Making use of (15), and taking into account the identity $\stackrel{\Gamma}{\nabla} \vartheta^{I} \equiv 0$ following from (3), (4) 
-with $\stackrel{\Gamma}{\nabla}$ as given by (A20)-, we separate equations (20) (22) into their real and imaginary parts as

$$
\begin{gathered}
\begin{aligned}
0 \approx \eta_{I} & =\nabla^{\Gamma} \eta_{I}+i \epsilon_{I J}{ }^{K} K^{J} \wedge \eta_{K} \\
& =i \vartheta_{I} \wedge \vartheta_{J} \wedge K^{J}
\end{aligned} \\
0 \approx \vartheta_{I} \wedge{ }^{\#} F^{I}=\vartheta_{I} \wedge{ }^{\#} \mathcal{R}^{I}+i \vartheta_{I} \wedge{ }^{\#} \nabla K^{I} \\
0 \approx \vartheta_{I} \wedge F^{I}=\vartheta_{I} \wedge \mathcal{R}^{I}-i d\left(\vartheta_{I} \wedge K^{I}\right)
\end{gathered}
$$

In analogy with the complex matrix $\Psi^{I J}$ in (23), we propose a real matrix $\Phi^{I J}$ such that

$$
\mathcal{R}^{I}=\Phi^{I J} \eta_{J}
$$

By introducing the notation

$$
\begin{aligned}
\Gamma^{I} & :=d \Gamma^{I}+\frac{1}{2} \epsilon^{I}{ }_{J K} \Gamma^{J} \wedge \Gamma^{K}, \\
{ }^{\#} S^{I} & :=\epsilon^{I}{ }_{J K} K^{J} \wedge K^{K},
\end{aligned}
$$

so that (13) decomposes into

$$
\mathcal{R}^{I}=\stackrel{\Gamma}{F^{I}}-\frac{1}{2} \# S^{I}
$$

From (33) with (30) we get

$$
S^{I}=2\left({ }^{\#}{ }^{\Gamma}{ }^{I}-\Phi^{I J} \vartheta_{J}\right)
$$

while on the other hand the inversion of (32) yields

$$
K^{I}=\#\left(\frac{1}{2 \sqrt{2 S}} \epsilon_{J K}^{I} S^{J} \wedge S^{K}\right),
$$

being $S$ the determinant $S:=\frac{1}{3 !} \eta^{a b c} \epsilon_{I J K} S_{a}^{I} S_{b}^{J} S_{c}^{K}$. The condition for both (27) and the imaginary part of (29) to vanish simultaneously is $\vartheta_{I} \wedge K^{I}=0$, which by invoking (35) gives rise to

$$
\left.0=\vartheta_{I} \wedge K^{I}=-\frac{1}{\sqrt{2 S}} \epsilon_{J K}^{I}\left(e_{I}\right\rfloor S^{J}\right) \# S^{K} .
$$

Replacing (34) into (36) taking into account that $\left.\epsilon_{J K}^{I}\left(e_{I}\right\rfloor^{\#} \stackrel{\Gamma}{F}^{J}\right)=-\#\left(\stackrel{\Gamma}{\nabla} \nabla \vartheta_{K}\right) \equiv 0$, condition (36) reduces to $\Phi_{[I J]}=0$. As a byproduct, the symmetric $\Phi_{I J}$ ensuring the vanishing of (27) and of the imaginary part of (29) also cancels the real part of (28), since the latter, with (30), reads $\vartheta_{I} \wedge{ }^{\#} \mathcal{R}^{I}=\Phi^{I J} \vartheta_{I} \wedge \vartheta_{J}$. On the other hand, the real part of (29) reduces to $\vartheta_{I} \wedge \mathcal{R}^{I}=\Phi^{I J} \vartheta_{I} \wedge \eta_{J}=\Phi_{I}^{I} \eta$, thus vanishing for $\Phi_{I}^{I}=0$. So, any symmetric traceless matrix $\Phi_{I J}$ solves the system (27)-(29) up to the imaginary part of the vector constraint (28).

\section{THREE-METRIC DEPENDENT SOLUTION FOR THE EXTRINSIC CURVATURE}

The approach proposed by us mainly differs from the previously considered ones in that we pay attention to the connections - that is, to the fundamental variablesrather than to the field strengths built with them. Actually, not the components of $F^{I}$ or of $\mathcal{R}^{I}$ as in previous section but those of the nonlinear boost connection $K^{I}$ (identical with the ADM extrinsic curvature) play the relevant role as the quantities to be determined.

Starting from the constraints in their form (27)- (29), we find that the Gauss law (27) is trivially fulfilled for the extrinsic curvature $\left.K_{I J}:=e_{I}\right\rfloor K_{J}$ a symmetric matrix. This result can alternatively be obtained from (6). Indeed, by replacing in it (2), due to the fact that the $\beta$-independent part of the covariant derivative vanishes identically, we get

$$
\nabla_{a} E_{I}^{a}=\beta \epsilon_{I J}^{M} K_{a}^{J} E_{M}^{a} \approx 0,
$$

which is automatically satisfied by any symmetric matrix $K_{M}^{J}:=K_{a}{ }^{J} e_{M}{ }^{a}$. As a corollary of $K_{[I J]}=0$, the real contribution of (28) automatically vanishes, as it is easily seen by explicitly displaying

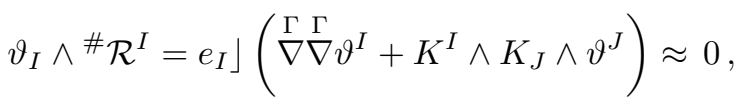

where $\Gamma^{\Gamma} \vartheta^{I}$ stands for the vanishing spatial contribution to torsion, while $\vartheta_{J} \wedge K^{J}$ is zero in view of the symmetry of $K_{I J}$. For the same reason, the imaginary contribution of (29) is also null. Consequently, Eqs. (28) and (29) reduce respectively to

$$
\begin{aligned}
0 & \approx \vartheta_{I} \wedge^{\#} F^{I}=i \vartheta_{I} \wedge^{\#} \stackrel{\Gamma}{\nabla} K^{I} \\
& =-\frac{i}{2} \epsilon^{I J K} e_{M}^{a} \nabla_{a}\left(K_{K}^{M}-\delta_{K}^{M} K_{N}^{N}\right) \vartheta_{I} \wedge \vartheta_{J}
\end{aligned}
$$

and

$$
\begin{aligned}
0 & \approx \vartheta_{I} \wedge F^{I}=\vartheta_{I} \wedge \mathcal{R}^{I} \\
& =-\frac{1}{2} \eta\left[{ }^{(3)} R-K_{I}{ }^{J} K_{J}{ }^{I}+K_{I}{ }^{I} K_{J}{ }^{J}\right]
\end{aligned}
$$

with ${ }^{(3)} R$ as the Riemannian scalar curvature built from the three-metric $q_{a b}:=\delta_{I J} e_{a}{ }^{I} e_{b}{ }^{J}$. Comparing (39), (40) with (24), (25), the explicit form of $\Psi^{[I J]}$ and $\Psi_{I}^{I}$ follows in terms of $K_{I J}$. The transition to the standard ADM formalism can be easily performed. Making use of (18) with (39), we calculate

$$
\begin{aligned}
\frac{i}{e} \mathcal{V}_{a} & =i \epsilon_{I J K} \Psi^{I J} e_{a}{ }^{K}=e_{a}{ }^{K} e_{M}{ }^{b} \stackrel{\Gamma}{b}_{b}\left(K_{K}{ }^{M}-\delta_{K}^{M} K_{N}{ }^{N}\right) \\
& =D_{b}\left(K_{a}{ }^{b}-\delta_{a}^{b} K_{c}{ }^{c}\right) \approx 0,
\end{aligned}
$$

being $K_{a b}:=K_{a}{ }^{I} e_{b I}$, while $D_{a}$ is the covariant derivative associated with the 3-metric $q_{a b}$, built with the ordinary 
Christoffel symbol (5). In the vector constraint written as (41) we recognize the standard form of the momentum constraint in ADM variables 25] with the extrinsic curvature playing the role of the momentum. On the other hand, from (19) with (40), and in view of the invariance of the expressions involved, one finds

$$
-\frac{1}{e^{2}} \mathcal{S}=-2 \Psi_{I}^{I}={ }^{(3)} R-K_{a}{ }^{b} K_{b}{ }^{a}+K_{a}{ }^{a} K_{b}{ }^{b} \approx 0
$$

So, from (41) and (42) we recover the standard ADM constraints [25], which for clarity we display separately as

$$
\begin{aligned}
D_{b}\left(K_{a}{ }^{b}-\delta_{a}^{b} \operatorname{tr} K\right) & \approx 0, \\
R-\operatorname{tr}\left(K^{2}\right)+(\operatorname{tr} K)^{2} & \approx 0 .
\end{aligned}
$$

(In (44) and in the following we use the simplified notation $R:={ }^{(3)} R$.) The variables concerned in (43) are the matrix $K_{a b}$ (in the following we refer to it simply as $K$ ) as much as the non explicitly displayed three-metric $q_{a b}$ present in the Christoffel symbols of the covariant derivative. Being $K$ symmetric, in each point of the spacetime manifold it is diagonalizable by means of an orthogonal transformation, so that its eigenvalues arranged in the diagonal matrix turn out to be the relevant variables, while the three degrees of freedom of the diagonalizing orthogonal matrix become absorbed into the three-metric and thus -let us say- geometrized. As it is well known, from a $3 \times 3$ matrix $K$ it is possible to build three invariants, namely its trace $\operatorname{tr} K$, the $\operatorname{trace} \operatorname{tr}\left(K^{2}\right)$ of the matrix square, and the determinant $\operatorname{det} K$, being such invariants related to the matrix $K$ by the characteristic equation

$$
K^{3}-(\operatorname{tr} K) K^{2}+\frac{1}{2}\left[(\operatorname{tr} K)^{2}-\operatorname{tr}\left(K^{2}\right)\right] K-I \operatorname{det} K=0,
$$

see [24]. Being the invariants expressible in terms of the matrix eigenvalues, from now on we pay attention to the invariants rather than to the eigenvalues.

One can diminish the number of invariants involved in (43), (44) by imposing the zero trace assumption $\operatorname{tr} K=$ 0 , the latter constituting the usual slicing condition used as a gauge fixing of the reparametrization invariance [25]. Accordingly, (44) reduces to

$$
\operatorname{tr}\left(K^{2}\right) \approx R
$$

thus determining a further invariant, namely $\operatorname{tr}\left(K^{2}\right)$, in terms of the scalar curvature; that is, in terms of a quantity built from the three-metric $q_{a b}$ of the underlying Riemannian space and from their derivatives up to second order. So, only det $K$ remains as an unknown function to be determined from the differential condition (43) in its simplified form

$$
D_{b} K_{a}{ }^{b} \approx 0
$$

which (for diagonalized $K$ ) relates the first derivatives of the remaining invariant $\operatorname{det} K$ with the invariant itself and with several geometrical objects involving the metric and its derivatives. As a result, as far as one primarily looks for solutions of $K$ being functionals of arbitrary metrics (other solutions will be considered below), $\operatorname{det} K$ has to be expressible as an invariant built with the metric tensor $q_{a b}$ and its derivatives up to third order. The reason for it is that the first derivative of the invariant $\operatorname{tr}\left(K^{2}\right)$, implicitly present in (47) through (46), yields a derived scalar curvature $R$, the latter comprising up to second derivatives of the metric.

Having in mind the previous discussion, let us follow an indirect way to find a matrix $K$ satisfying all the required features. First of all we parametrize such matrix as

$$
K^{a b}=\eta^{a c d} D_{c} S_{d}^{b}
$$

in terms of the (derived) matrix $S_{d}{ }^{b}$ to be determined. The latter matrix must be chosen so that it guarantees that, as studied above, $K^{a b}$ is symmetric and traceless, satisfying in addition equation (47). From (48) follows

$$
K^{a b} \eta_{a b c}=D_{b}\left(S_{c}^{b}-\delta_{c}^{b} S_{d}^{d}\right)
$$

showing that the symmetry condition on (48) requires the vanishing of the r.h.s. of (49). On the other hand, (48) is trivially traceless provided $S_{d b}$ is a symmetric matrix. Finally, we find the divergence of (48) to be

$$
D_{b} K_{a}{ }^{b}=\eta_{a}{ }^{b c} R_{b d} S_{c}{ }^{d}+\eta_{a}{ }^{b c} D_{b} D_{d} S_{c}{ }^{d},
$$

where we made use of the formula (51) below, holding in three dimensions. Due to the covariance of $K$, we make the ansatz -in the particular case considered here that $K$ is taken to be a functional of an arbitrary three-metric $q_{a b}$ and of at most its third derivatives- that the matrix $S_{d}{ }^{b}$ in (48) has to be built from the Riemann tensor and its contractions. We know that in three dimensions no Weyl tensor exists [26], so that the Riemann tensor $R_{c b d} f:=$ $2\left(\partial_{[c} \Gamma_{b] d}^{f}+\Gamma_{[c h}^{f} \Gamma_{b] d}{ }^{h}\right)$ can be decomposed in terms of the Ricci tensor $R_{c d}:=R_{f c d} f$ and of the scalar curvature $R:=g^{c d} R_{c d}$ as

$$
R_{c b d}^{f}=-2 q_{d[c} R_{b]}^{f}+2 \delta_{[c}^{f} R_{b] d}-\delta_{[c}^{f} q_{b] d} R .
$$

In particular we choose for $S_{d}^{b}$ the combination

$$
S_{d}^{b}=k R_{d}^{b}+k^{\prime} \delta_{d}^{b} R
$$

which is symmetric as required and satisfies (50) automatically (recall the identity $D_{d} G_{c}{ }^{d} \equiv 0$, with $G_{c}{ }^{d}$ as the Einstein tensor). The remaining condition of vanishing (49) enforces us to fix $k^{\prime}=-k / 4$, so that (48) with (52) transforms into

$$
K^{a b}=k \eta^{a c d} D_{c}\left(R_{d}^{b}-\frac{1}{4} \delta_{d}^{b} R\right),
$$

satisfying all the requirements stipulated for $K$. In the r.h.s. of (53) we recognize the Cotton-York tensor (also 
called Bach tensor) built from the Cotton tensor in 3dimensional space, see eq.(97) of [27]. The five degrees of freedom of the symmetric traceless exterior curvature thus become expressed -through the five independent components of the Cotton-York tensor- in terms of any three-metric $q_{a b}$ without additional integrability conditions.

Notice that (46) is not completely foreign to our main result (53). Actually -as we will immediately see- other solutions are possible for the divergence condition (47). In the discussion preceding our concrete proposal, it was precisely (46) that played a crucial role in justifying to consider a contribution to $K$ built exclusively as a functional of an arbitrary three-metric, by reducing the invariant function $\operatorname{tr}\left(K^{2}\right)$ to the $q_{a b}$-dependent scalar curvature. (Recall that by also fixing $\operatorname{tr} K=0$, the only remaining invariant det $K$ and its derivatives became related solely to functions of the three-metric.) Thus, the general validity of (53) rests on having taken simultaneously into account (46) as a consistence condition. In other words, (53) can be taken as a general metric dependent solution as far as it refers to the system (47), (46) rather than merely to (47).

Observe that for three-metrics such that $R=0$, no independent dynamics for $K_{a b}$ exists any more if one requires the eigenvalues of $K$ to be real. Indeed, with $R=0$, (46) implies $\operatorname{tr}\left(K^{2}\right)=0$. Having already assumed $\operatorname{tr} K=0$, the characteristic equation (45) reduces to

$$
K^{3}-I \operatorname{det} K=0 .
$$

The discriminant involved in the solution of (54) is positive, so that the eigenvalues of $K$ consist of one real and two complex conjugate roots, thus leaving $K_{a b}=0$ as the only physically admissible extrinsic curvature.

\section{ADDITIONAL SOLUTIONS OF $K_{a b}$}

The natural question to be asked now is about the existence of nontrivial $K$ 's which, although necessarily linked to a metric with $R \neq 0$, are nevertheless irreducible to the functional (53) of such metric. We are going to prove that, in fact, (53) does not exhaust all possible solutions of the constraints (46) and (47).

Due to the interplay existing between $K$ and the threemetric, it is illustrative to consider isotropic metrics, for which (53) vanish, guaranteeing that any possible $K \neq$ 0 must be independent of (53). The general isotropic metric is given by its nonvanishing components

$$
q_{11}=\frac{1}{\phi}, \quad q_{22}=r^{2}, \quad q_{33}=r^{2} \sin ^{2} \theta,
$$

where $\phi=\phi(r)$. The corresponding components of the Christoffel connection (5) read

$$
\Gamma_{11}^{1}=-\frac{\partial_{r} \phi}{2 \phi}, \quad \Gamma_{22}^{1}=-r \phi, \quad \Gamma_{33}^{1}=-r \phi \sin ^{2} \theta,
$$

$$
\begin{array}{ll}
\Gamma_{12}^{2}=\Gamma_{21}^{2}=\frac{1}{r}, & \Gamma_{33}^{2}=-\sin \theta \cos \theta, \\
\Gamma_{13}^{3}=\Gamma_{31}^{3}=\frac{1}{r}, & \Gamma_{23}^{3}=\Gamma_{32}^{3}=\cot \theta,
\end{array}
$$

the Ricci tensor $R_{a b}:=2\left(\partial_{[a} \Gamma_{c] b}^{c}+\Gamma_{[a d}^{c} \Gamma_{c] b}^{d}\right)$ calculated from (56) is diagonal, with

$$
\begin{aligned}
R_{11} & =\frac{\partial_{r} \phi}{r \phi}, \\
R_{22} & =\frac{1}{2 r} \partial_{r}\left[r^{2}(\phi-1)\right], \\
R_{33} & =\frac{\sin ^{2} \theta}{2 r} \partial_{r}\left[r^{2}(\phi-1)\right],
\end{aligned}
$$

and the scalar curvature reads

$$
R:=q^{a b} R_{a b}=\frac{2}{r^{2}} \partial_{r}[r(\phi-1)] .
$$

It is trivial to check, by replacing (57) and (58), that the solution (53) of $K_{a b}$ reducible to the three-metric vanishes for any isotropic metric.

In order to simplify calculations, let us rewrite the matrix $K_{a}{ }^{b}$ (with general indices) as

$$
K_{a}{ }^{b}=e_{a}{ }^{M} K_{M}{ }^{N} e^{b}{ }_{N},
$$

in terms of the symmetric matrix with internal indices $K_{M}{ }^{N}$, which can be diagonalized with the help of an orthogonal matrix $O_{M}{ }^{I}$ as $K_{M}{ }^{N}=O_{M}{ }^{I} \Delta_{I}{ }^{J} O^{N}{ }_{J}$ with $\Delta_{I}^{J}$ diagonal. We write (59) as

$$
K_{a}{ }^{b}=\hat{e}_{a}^{I} \Delta_{I}^{J} \hat{e}^{b}{ }_{J}
$$

by introducing the redefined dreibein $\hat{e}_{a}^{I}:=e_{a}{ }^{M} O_{M}{ }^{I}$. Accordingly, (47) build with (15) transforms into

$$
\left.\left.0=D_{b} K_{a}{ }^{b}=\partial_{b}\right\rfloor D K_{a}{ }^{b}=\left(\hat{e}_{I}\right\rfloor \hat{D} \Delta_{J}^{I}\right) \hat{e}_{a}{ }^{J},
$$

where the relation (4) was taken into account, being

$$
\left.\left.\left.\hat{\Gamma}_{I J}:=\hat{e}_{[I}\right\rfloor d \hat{\vartheta}_{J]}-\frac{1}{2}\left(\hat{e}_{I}\right\rfloor \hat{e}_{J}\right\rfloor d \hat{\vartheta}^{K}\right) \hat{\vartheta}_{K},
$$

with $\hat{\vartheta}^{I}:=d x^{a} \hat{e}_{a}^{I}$, and

$$
\hat{D} \Delta_{J}^{I}:=d \Delta_{J}^{I}+\hat{\Gamma}_{K}^{I} \Delta_{J}^{K}-\hat{\Gamma}_{J}{ }^{K} \Delta_{K}{ }^{I} .
$$

In the following we fix the gauge of the $S O(3)$ symmetry by choosing $O_{M}{ }^{I}=\delta_{M}^{I}$, so that $\hat{e}_{a}{ }^{I}=e_{a}{ }^{I}$. (Accordingly, from now on we suppress the hat over the triads as much as over the connections.)

Eq. (61) shows the correspondence between the alternative notations in terms of the three-metric or of triads respectively. Then, instead of the metric components (55) and the ordinary Christoffel symbols (56), one can introduce the triads

$$
\vartheta^{r}=\frac{d r}{\sqrt{\phi}}, \quad \vartheta^{\theta}=r d \theta, \quad \vartheta^{\varphi}=r \sin \theta d \varphi
$$


and the corresponding connection 1-form (62), with antisymmetric components

$$
\Gamma_{r \theta}=\frac{\sqrt{\phi}}{r} \vartheta^{\theta}, \quad \Gamma_{r \varphi}=\frac{\sqrt{\phi}}{r} \vartheta^{\varphi}, \quad \Gamma_{\theta \varphi}=\frac{\cot \theta}{r} \vartheta^{\varphi} .
$$

Making use of (64) and (65), we find the explicit form of (61) by calculating

$$
\begin{aligned}
\left.e_{I}\right\rfloor D \Delta_{r}{ }^{I} & =\frac{\sqrt{\phi}}{r}\left[\partial_{r}\left(r^{2} \Delta_{r}^{r}\right)-r\left(\Delta_{\theta}{ }^{\theta}+\Delta_{\varphi}{ }^{\varphi}\right)\right](66) \\
\left.e_{I}\right\rfloor D \Delta_{\theta}{ }^{I} & =\frac{1}{r}\left[\partial_{\theta} \Delta_{\theta}{ }^{\theta}+\cot \theta\left(\Delta_{\theta}{ }^{\theta}-\Delta_{\varphi}^{\varphi}\right)\right], \\
\left.e_{I}\right\rfloor D \Delta_{\varphi}{ }^{I} & =\frac{1}{r \sin \theta} \partial_{\varphi} \Delta_{\varphi}^{\varphi}
\end{aligned}
$$

We propose the suitable parametrization

$$
\begin{aligned}
\Delta_{r}{ }^{r} & =\lambda \cos \omega, \\
\Delta_{\theta}{ }^{\theta} & =-\frac{\lambda}{2}(\cos \omega-\sqrt{3} \sin \omega), \\
\Delta_{\varphi}^{\varphi} & =-\frac{\lambda}{2}(\cos \omega+\sqrt{3} \sin \omega),
\end{aligned}
$$

for a (diagonalized) real traceless matrix, in terms of which equations (61) with (66) - 68) transform into

$$
\begin{aligned}
& 0=\partial_{r}\left(r^{3} \lambda \cos \omega\right) \\
& 0=\lambda\left[\partial_{\theta} \cos \omega-\frac{\sqrt{3}}{\sin ^{2} \theta} \partial_{\theta}\left(\sin \omega \sin ^{2} \theta\right)\right] \\
& 0=\lambda \partial_{\varphi}(\cos \omega+\sqrt{3} \sin \omega) .
\end{aligned}
$$

The choice (69)-(71) has the virtue of guaranteeing automatically $\operatorname{tr} K=0$, while $\operatorname{det} K=$ $\frac{1}{4} \lambda^{3} \cos \omega\left(1-4 \sin ^{2} \omega\right)$ and $\operatorname{tr}\left(K^{2}\right)=\frac{3}{2} \lambda^{2}$. Replacing the last expression as much as (58) into (46), we get

$$
\frac{3}{2} \lambda^{2}=\frac{2}{r^{2}} \partial_{r}[r(\phi-1)],
$$

implying that $\lambda$ exclusively depends on $r$.

Let us first consider the case $R=0$, noting that we are working with a metric different from the trivial Euclidean one. As read out from (58) and (75), $R=0$ corresponds to $\lambda=0$, so that (72)-(74) are trivially fulfilled. On the other hand, (75) reduces to $\partial_{r}[r(\phi-1)]=0$, whose solution reads

$$
r(\phi-1)=-2 m
$$

with the integration constant suitably denoted as a mass in order to reproduce the well known Schwarzshild function solution in (55) or (64). We read it out from (76) to be

$$
\phi=1-\frac{2 m}{r} .
$$

As discussed above, $K_{a b}=0$. No $K$ exists in this case, neither as (53) nor as a different -metric-independentcontribution.
Less trivial is the case with $R \neq 0$, and thus with $\lambda \neq 0$. Certainly, (53) vanishes as for any isotropic three-metric. However, a nontrivial $K$ not reducible to (53) can be found as follows. By expressing (73) in the form

$$
0=\sin \omega \partial_{\theta} \omega+\sqrt{3}\left(\partial_{\theta} \sin \omega+2 \sin \omega \cot \theta\right),
$$

it is clear that it is satisfied for $\sin \omega=0$. This is the case we are going to consider.

But let us briefly show before what happens for $\sin \omega \neq$ 0 . By rewriting (72) and (73) respectively as

$$
\partial_{r} \omega=\cot \omega \partial_{r} \log \left(r^{3} \lambda\right),
$$

and

$$
\partial_{\theta} \omega=-\frac{2 \sqrt{3} \cot \theta}{(1+\sqrt{3} \cot \omega)},
$$

from the integrability condition $\partial_{\theta} \partial_{r} \omega=\partial_{r} \partial_{\theta} \omega$ we find $\tan \omega=-2 \sqrt{3}$, implying $\cos \theta=0$, whose consistence with (74) is guaranteed since $\omega$ is a constant, so that $\partial_{\varphi} \omega=0$. The physical meaning of this result will be studied elsewhere.

We concentrate now on the case with $\sin \omega=0$. The discriminant of the cubic equation (45) with $\operatorname{tr} K=0$, that is of

$$
K^{3}-\frac{1}{2} \operatorname{tr}\left(K^{2}\right) K-I \operatorname{det} K=0,
$$

is found to be proportional to $\sin \omega$ as

$$
\begin{aligned}
\operatorname{discrim} & =\left(\frac{\operatorname{det} K}{2}\right)^{2}-\left(\frac{\operatorname{tr}\left(K^{2}\right)}{6}\right)^{3} \\
& =\frac{\lambda^{6}}{64} \sin ^{2} \omega\left[2 \cos ^{2} \omega\left(\sin ^{2} \omega-4\right)-1\right]
\end{aligned}
$$

Accordingly, condition $\sin \omega=0$ forces the solutions of (81) to be three real eigenvalues summing zero, being two of them equal to each other, as displayed in (87)-899) below. In fact, the nontrivial -although very simpledynamics for $K$ consists of equation (72) reduced to

$$
0=\partial_{r}\left(r^{3} \lambda\right)
$$

while (73) and (74) are trivially satisfied. We solve (83) as

$$
\lambda=\frac{2 k_{0}}{r^{3}}
$$

in terms of the arbitrary constant $2 k_{0}$. The value (84) is to be replaced on the one hand in (75), yielding

$$
\partial_{r}\left[r(\phi-1)+\frac{k_{0}^{2}}{r^{3}}\right]=0,
$$

giving rise, in analogy to (77), to the generalized Schwarzshild solution

$$
\phi=1-\frac{2 m}{r}-\frac{k_{0}^{2}}{r^{4}}
$$


and on the other hand (84), when replaced in (69)-(71) with $\sin \omega=0$, yields the eigenvalues

$$
\begin{aligned}
\Delta_{r}{ }^{r} & =\lambda=\frac{2 k_{0}}{r^{3}}, \\
\Delta_{\theta}{ }^{\theta} & =-\frac{\lambda}{2}=-\frac{k_{0}}{r^{3}}, \\
\Delta_{\varphi}{ }^{\varphi} & =-\frac{\lambda}{2}=-\frac{k_{0}}{r^{3}},
\end{aligned}
$$

which determine a nontrivial extrinsic curvature not reducible to (53). Recall that this is the main result we wanted to derive in the present paragraph. It is worth noticing that, according to (60) with the choice (64) of triads we made, we can express the components of $K_{a}{ }^{b}$ in terms of (87) - (89) as

$$
\begin{aligned}
& K_{1}{ }^{1}=e_{1}{ }^{r} \Delta_{r}{ }^{r} e_{r}{ }^{1}=\Delta_{r}{ }^{r}=\frac{2 k_{0}}{r^{3}}, \\
& K_{2}{ }^{2}=e_{2}{ }^{\theta} \Delta_{\theta}{ }^{\theta} e_{\theta}{ }^{2}=\Delta_{\theta}{ }^{\theta}=-\frac{k_{0}}{r^{3}}, \\
& K_{3}{ }^{3}=e_{3}{ }^{\varphi} \Delta_{\varphi}{ }^{\varphi} e_{\varphi}{ }^{3}=\Delta_{\varphi}{ }^{\varphi}=-\frac{k_{0}}{r^{3}},
\end{aligned}
$$

completing the solution provided by the metric (55) with (86).

\section{CONCLUSIONS}

Our main result establishes that any possible solution for the extrinsic curvature $K_{a b}$ as found out from (46) and (47) is defined up to a contribution of the form (53), involving the Cotton-York tensor with the proportionality constant $k$. Thus, the total vanishing of $K_{a b}$ requires the Cotton-York tensor to vanish, as it is the case for general isotropic metrics. Recall the relation existing between $K$ and the time derivative of the three-metric [25], namely

$$
K_{a b}=\frac{1}{N}\left(D_{(a} N_{b)}-\frac{1}{2} \partial_{\tau} q_{a b}\right),
$$

(with $N$ as the lapse function and $N_{a}$ as the shift vector), showing an interplay between either vanishing or not vanishing $K$, and the corresponding either static or time-evolving metric.

The Cotton tensor $D_{[c}\left(R_{d]}^{b}-\frac{1}{4} \delta_{d]}^{b} R\right)$ present in (53) is conformally invariant under rescalings of the 3-metric $q_{a b} \rightarrow \tilde{q}_{a b}=\varphi(x) q_{a b}$. Although originally expressed as a functional of the three-metric $q_{a b}$, (53) may be alternatively formulated in different forms, for instance as

$$
K^{I}=k^{\#} \stackrel{\Gamma}{\nabla}\left[{ }^{*}{ }^{I}-\frac{1}{2} \vartheta^{I} \#\left(\vartheta_{J} \wedge F^{\Gamma}\right)\right],
$$

in terms of (31), or equivalently as

$$
\left.\left.\left.K^{I}=k^{\#} \hat{D}\left(e_{J}\right\rfloor \hat{R}^{I J}-\frac{1}{4} \vartheta^{I} e_{M}\right\rfloor e_{N}\right\rfloor \hat{R}^{M N}\right),
$$

with the covariant differential built with connection (62) -related to $\Gamma^{I}$ as (A10)- and with the corresponding curvature 2-form $\hat{R}_{I}^{J}:=d \hat{\Gamma}_{I}^{J}+\hat{\Gamma}_{K}{ }^{J} \wedge \hat{\Gamma}_{I}{ }^{K}$ related to (31) as $\stackrel{\Gamma}{F}^{I}=\frac{1}{2} \epsilon^{I}{ }_{J K} \hat{R}^{J K}$.

We have shown that the search for a general extrinsic curvature $K_{a b}$ going beyond (53) is enabled by diagonalizing $K$ in such a way that its eigenvalues play the role of dynamical variables. Certainly, both quantities $K_{a b}$ and $q_{a b}$ are mutually linked to each other. However, no general solutions $K=K(q)$ or $q=q(K)$ exist. The interplay between $K_{a b}$ and $q_{a b}$ rather manifest itself twofold. On the one hand, given a particular metric, restrictions result on the eigenvalues of $K$. For instance, for the isotropic metric with $R=0$ we found $K=0$ (Schwarzschild static solution), whereas with $R \neq 0$ we obtained a certain $K \neq 0$, related through (93) to the time evolution of the three-metric. Obviously, this procedure to solve the eigenvalues of $K$ is easily generalizable. Reciprocally, given $K$, the metric of course is not entirely fixed by it, but the metric features are affected by $K$ to some extent. Recall for instance (86) as the modification of (77) induced by (87)- 89). In general, integrability conditions impose restrictions on the metric components, regarding their functional form or at least their coordinate dependence.

\section{Acknowledgments}

The authors are very grateful to Profs. Jaime Julve, Jesús Martín and Friedrich W. Hehl for useful discussions and remarks. The work of one of us (R.T.) was supported by CSIC and CESGA.

\section{APPENDIX A: NONLINEAR PGT CONNECTIONS}

Ordinary spacetime geometry, characterized by both reference frames and connections, can be derived as a gauge theory of the Poincaré group realized nonlinearly, as discussed in 18]. Given the linear Poincaré connections, consisting of the Lorentz and translational contributions $\stackrel{L o r}{\Gamma_{\alpha}^{\beta}}$ and $\stackrel{(T)}{\Gamma^{\alpha}}$ respectively, transforming as

$$
\delta \Gamma_{\alpha}^{\text {Lor }}=\stackrel{\text { Lor }}{D} \zeta_{\alpha}^{\beta}, \quad \delta \Gamma^{\alpha}=-\zeta_{\beta}^{\alpha} \Gamma^{\beta}+\stackrel{\text { Lor }}{D} \epsilon^{\alpha},
$$

(where the abbreviation Lor over the covariant differentials in (A1) indicates that they are constructed with the linear Lorentz connection $\stackrel{L o r}{\Gamma^{\alpha \beta}}$ ) it is characteristic for the nonlinear approach to provide us with auxiliary Goldstone-like translational fields $\xi^{\alpha}$ transforming as coordinates, namely

$$
\delta \xi^{\alpha}=-\zeta_{\beta}^{\alpha} \xi^{\beta}-\epsilon^{\alpha} .
$$


(Greek letters $\alpha, \beta \ldots$ denote internal Lorentz indices running from 0 to 3.) Translational connections modified with the help of the fields $\xi^{\alpha}$ as the nonlinear translational connections

$$
\vartheta^{\alpha}:=\stackrel{\operatorname{Lor}}{D} \xi^{\alpha}+\stackrel{(T)}{\Gamma}
$$

transform as Lorentz covectors, that is

$$
\delta \vartheta^{\alpha}=-\zeta_{\beta}{ }^{\alpha} \vartheta^{\beta}
$$

We identify A4 as Lorentz coframe 1-forms.

On the other hand, the nonlinear approach also enables us to deal with $S O(3)$ tensors and connections instead of with Lorentz ones, if desired 1] 2]. Indeed, by decomposing the Lorentz generators $L_{\alpha \beta}$ into space rotations $S_{I}$ and boosts $B_{I}$, defined respectively as

$$
S_{I}:=-\epsilon_{I}^{J K} L_{J K} \quad, \quad B_{I}:=2 L_{I 0}, \quad(I=1,2,3),
$$

the components of the nonlinear Poincaré connection on the corresponding Lie algebra read

$$
\widetilde{\Gamma}=-i \hat{\vartheta}^{0} P_{0}-i \hat{\vartheta}^{I} P_{I}+i \Gamma^{I} S_{I}+i K^{I} B_{I},
$$

where the nonlinear connections are defined with the help of boost related Goldstone fields $\beta^{I}$. Exactly as the translational Goldstone fields $\xi^{\alpha}$ are coordinate-like fields, the boost Goldstone fields $\beta^{I}$ are velocity-like fields, being rearrangeable into the components of a Lorentz four-vector $\left(\gamma, \gamma \beta^{I}\right)$, with $\gamma:=1 / \sqrt{1-\beta^{2}}$. In terms of them we build the boost-transformation-analogous matrix

$$
\begin{aligned}
b_{0}{ }^{0} & =\left(b^{-1}\right)_{0}{ }^{0}:=\gamma, \\
b_{0}{ }^{I} & =-\left(b^{-1}\right)_{0}{ }^{I}:=-\gamma \beta^{I}, \\
b_{I}{ }^{0} & =-\left(b^{-1}\right)_{I}{ }^{0}:=-\gamma \beta_{I}, \\
b_{J}{ }^{I} & =\left(b^{-1}\right)_{J}{ }^{I}:=\delta_{J}^{I}+(\gamma-1) \frac{\beta_{J} \beta^{I}}{\beta^{2}} .
\end{aligned}
$$

With the help of A7 we define he nonlinear Poincaré connections in (A6) as

$$
\begin{aligned}
& \hat{\vartheta}^{0}=\vartheta^{\alpha} b_{\alpha}{ }^{0}, \\
& \hat{\vartheta}^{I}=\vartheta^{\alpha} b_{\alpha}{ }^{I}, \\
& \Gamma^{I}:=\frac{1}{2} \epsilon_{J K}^{I} \hat{\Gamma}^{J K} \\
& =\frac{1}{2} \epsilon_{J K}^{I}\left(b^{-1}\right)^{J \mu}\left({\left.\stackrel{L}{\Gamma}{ }_{\mu}{ }^{\nu} b_{\nu}{ }^{K}-d b_{\mu}{ }^{K}\right)}\right),
\end{aligned}
$$

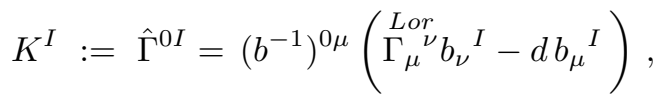

with $\vartheta^{\alpha}$ in (A8) and (A9) given by (A3). Formally, A8 (A11) are analogous to gauge transformations, with the main difference that (A7) are Goldstone fields of the theory rather than group parameters. Thus (A8)-(A11) are definitions of new variables whose variations are easily checked to be

$$
\delta \hat{\vartheta}^{0}=0
$$

$$
\begin{aligned}
\delta \hat{\vartheta}^{I} & =\epsilon_{J K}^{I} \boldsymbol{\Theta}^{J} \hat{\vartheta}^{K}, \\
\delta K^{I} & =\epsilon^{I}{ }_{J K} \boldsymbol{\Theta}^{J} K^{K}, \\
\delta \Gamma^{I} & =-\nabla \boldsymbol{\Theta}^{I} \\
& :=-\left(d \boldsymbol{\Theta}^{I}+\epsilon_{J K}^{I} \Gamma^{J} \boldsymbol{\Theta}^{K}\right),
\end{aligned}
$$

corresponding to $S O(3)$ transformations with the group parameter $\Theta^{a}$ built from $\zeta_{\beta}{ }^{\alpha}$ in A2 with the help of the velocity fields as

$$
\Theta^{I}:=-\frac{1}{2} \epsilon^{I}{ }_{J K}\left[\zeta^{J K}+\frac{2 \gamma}{(\gamma+1)} \zeta^{0 J} \beta^{K}\right] .
$$

Notwithstanding, although not explicitly displayed, the total symmetry remains the Poincaré one. Notice that the tetrads become split into an $S O(3)$ singlet $\hat{\vartheta}^{0}$ plus an $S O(3)$ covector $\hat{\vartheta}^{I}$, while the Lorentz connection decomposes into a nonlinear boost connection 1-form $K^{I}$ transforming as a $S O(3)$ covector plus a $S O(3)$ connections $A^{I}$.

The nonlinear field strength built from (A6) reads

$d \widetilde{\Gamma}+\widetilde{\Gamma} \wedge \widetilde{\Gamma}=-i \hat{T}^{0} P_{0}-i \hat{T}^{I} P_{I}+i \mathcal{R}^{I} S_{I}+i\left(\stackrel{\Gamma}{\nabla} K^{I}\right) B_{I}$

which plays the role of the general Poincaré curvature, where the explicit form of $\mathcal{R}^{I}$ and $\nabla^{\Gamma} K^{I}$ does not differ from (13) and (14) respectively, while the torsion components are defined as the $S O(3)$ quantities

$$
\begin{aligned}
& \hat{T}^{0}:=d \hat{\vartheta}^{0}+\hat{\vartheta}_{I} \wedge K^{I}, \\
& \hat{T}^{I}:=\Gamma \hat{\vartheta}^{I}+\hat{\vartheta}^{0} \wedge K^{I},
\end{aligned}
$$

with

$$
\stackrel{\Gamma}{\nabla} \hat{\vartheta}^{I}:=d \hat{\vartheta}^{I}+\epsilon_{J K}^{I} \Gamma^{J} \wedge \hat{\vartheta}^{K}
$$

Vanishing torsion implies that the Lorentz connection reduces to the (anholonomic) Christoffel connection

$$
\begin{aligned}
\Gamma_{\alpha \beta}^{\{\}} & \left.\left.\left.:=e_{[\alpha}\right\rfloor d \vartheta_{\beta]}-\frac{1}{2}\left(e_{\alpha}\right\rfloor e_{\beta}\right\rfloor d \vartheta^{\gamma}\right) \vartheta_{\gamma} \\
& =-d x^{i} e_{[\alpha}^{j}\left(\partial_{i} e_{j \beta]}-\Gamma_{i j}{ }^{k} e_{k \beta]}\right),
\end{aligned}
$$

(with small latin letters $i, j \ldots$ of the middle of the alphabet assigned to the general spacetime coordinate indices of the underlying 4-dimensional manifold). In (A21 we recognize the relation to the usual Riemannian language of General Relativity involving the ordinary holonomic Christoffel symbol

$$
\Gamma_{i j}^{k}:=\frac{1}{2} g^{k l}\left(\partial_{i} g_{l j}+\partial_{j} g_{l i}-\partial_{l} g_{i j}\right)
$$

whose restriction to spatial slices is considered in the main body of the present paper. 
[1] A. Lopez-Pinto, A. Tiemblo and R. Tresguerres, Class. Quant. Grav. 14 (1997) 549, gr-qc/9603023

[2] A. Tiemblo and R. Tresguerres, Recent Res. Devel. Physics, Transworld Research Network, 5 (2004) 1255.

[3] A. Ashtekar, A. P. Balachandran and S. Jo, Int. J. Mod. Phys. A4 (1989) 1493.

[4] B. S. DeWitt, Phys. Rev. 160 (1967) 1113.

[5] J. F. Barbero, Phys. Rev. D51 (1995) 5507, gr-qc/9410014

[6] G. Immirzi, Nucl. Phys. Proc. Suppl. 57 (1997) 65, gr-qc/9701052

[7] J. Samuel, Class. Quant. Grav. 17 (2000) L141, gr-qc/0005095

[8] P. A. M. Dirac, Canadian Jour. of Math. 2 (1950) 129.

[9] A. Ashtekar, Phys. Rev. Lett. 57 (1986) 2244.

[10] A. Ashtekar, Phys. Rev. D36 (1987) 1587.

[11] T. Jacobson and L. Smolin, Class. Quant. Grav. 5 (1988) 583.

[12] R. P. Wallner, Phys. Rev. D42 (1990) 441.

[13] R. P. Wallner, Phys. Rev. D46 (1992) 4263.

[14] F. W. Hehl, P. Von Der Heyde, G. D. Kerlick and J. M. Nester, Rev. Mod. Phys. 48 (1976) 393.

[15] F. Gronwald and F. W. Hehl, (1995), gr-qc/9602013
[16] F. W. Hehl, J. D. McCrea, E. W. Mielke and Y. Neeman, Phys. Rept. 258 (1995) 1, gr-qc/9402012

[17] R. Tresguerres and E. W. Mielke, Phys. Rev. D62 (2000) 044004, gr-qc/0007072

[18] R. Tresguerres, Phys. Rev. D66 (2002) 064025.

[19] A. Randono, (2005), gr-qc/0504010

[20] H. Kodama, Phys. Rev. D42 (1990) 2548.

[21] A. Ashtekar, J. C. Baez and K. Krasnov, Adv. Theor. Math. Phys. 4 (2000) 1, gr-qc/0005126

[22] R. Capovilla, T. Jacobson and J. Dell, Phys. Rev. Lett. 63 (1989) 2325.

[23] J. F. Barbero, Class. Quant. Grav. 12 (1995) L5, gr-qc/9411016

[24] R. Capovilla, T. Jacobson and J. Dell, Class. Quant. Grav. 8 (1991) 59.

[25] E. Alvarez, Rev. Mod. Phys. 61 (1989) 561.

[26] S. Weinberg, Gravitation and Cosmology: Principles and Applications of the General Theory of Relativity (John Wiley \& Sons, New York, 1972).

[27] A. García, F.W. Hehl, C. Heinicke and A. Macías, Class. Quant. Grav. 21 (2004) 1099, gr-qc/0309008 